\title{
ON BREAKDOWN OF SOLUTIONS TO THE FULL COMPRESSIBLE NAVIER-STOKES EQUATIONS*
}

\author{
XIANGDI HUANG ${ }^{\dagger}$ AND JING LI ${ }^{\ddagger}$
}

\begin{abstract}
In this paper, when the initial density is away from vacuum, we establish a blow up criterion for the strong solutions of the viscous heat-conductive flows just in terms of the gradients of the velocity and the temperature, analogous to the Beal-Kato-Majda criterion for the ideal incompressible flow. In particular, the viscous coefficients $\mu$ and $\lambda$ are only required to satisfy the physical restrictions.
\end{abstract}

Key words. Breakdown, full compressible Navier-Stokes.

AMS subject classifications. 35Q30, 76N10

1. Introduction. This paper is devoted to studying the following 3D full compressible Navier - Stokes equations:

$$
\left\{\begin{array}{l}
\partial_{t} \rho+\operatorname{div}(\rho u)=0, \\
\partial_{t}(\rho u)+\operatorname{div}(\rho u \otimes u)-\mu \triangle u-(\mu+\lambda) \nabla(\operatorname{div} u)+\nabla P=0 \\
c_{v}\left[\partial_{t}(\rho \theta)+\operatorname{div}(\rho \theta u)\right]-\kappa \triangle \theta+P \operatorname{div} u=\frac{\mu}{2}\left|\nabla u+\nabla u^{T}\right|^{2}+\lambda(\operatorname{div} u)^{2},
\end{array}\right.
$$

where $\rho \geq 0$ denotes the density of the mass; $u$ is the velocity;

$$
P=R \rho \theta \quad(R>0)
$$

is the pressure; $\mu, \lambda, R, c_{v}$ and $\kappa$ are the physical constants satisfying $\mu>0, \lambda+\frac{2 \mu}{3} \geq$ $0, R>0, c_{v}>0$ and $\kappa>0$.

Let $\Omega$ be a bounded smooth domain in $R^{3}$. We consider an initial boundary value problem for $(1.1)-(1.3)$ with the following boundary conditions

$$
\begin{gathered}
\left.(\rho, u, \theta)\right|_{t=0}=\left(\rho_{0}, u_{0}, \theta_{0}\right) \text { in } \Omega \\
\left.u\right|_{\partial \Omega}=0,\left.\frac{\partial \theta}{\partial \nu}\right|_{\partial \Omega}=0
\end{gathered}
$$

where $\nu$ is the normal to $\partial \Omega$.

There are huge literatures on the studies of the well-posedness and behavior of solutions to (1.1). In the case that the density is away from vacuum, the one-dimensional problem was addressed by Kazhikhov and Shelukhin [26] for sufficient smooth data, and by Serre $[33,34]$ and Hoff [18] for discontinuous initial data. The global existence of classical solutions to the compressible Navier-Stokes equations in multidimensional case was obtained by Matsumura and Nishida [31] as long as the initial data is a small perturbation of a non-vacuum constant state in $H^{3}$. This result was generalized to

\footnotetext{
*Received December 11, 2009; accepted for publication January 11, 2010.

${ }^{\dagger}$ Department of Mathematics, University of Science and Technology of China, Hefei 230026, P. R. China; The Institute of Mathematical Sciences, The Chinese University of Hong Kong, Hong Kong (xdhuang@ustc.edu.cn).

${ }^{\ddagger}$ Institute of Applied Mathematics, AMSS, Academia Sinica, Beijing 100190, P. R. China; The Institute of Mathematical Sciences, The Chinese University of Hong Kong, Hong Kong (ajingli@gmail.com).
} 
the data with discontinuities by Hoff in a series of papers (see [18-20] and references therein). Furthermore, it is shown by Xin [37] that there is no global in time regular solution in $R^{3}$ to the compressible Naiver-Stokes equations (1.1) provided that the initial density is compactly supported.

In contrast to the theory of weak solutions for the incompressible Navier-Stokes system established by Leray [27], a major breakthrough for the compressible isentropic Navier-Stokes system is due to Lions. In [28,29], for $P(\rho)=a \rho^{\gamma}(a>0)$, Lions showed the global existence of weak solutions to the problem (1.1) provided that $\gamma \geq \frac{9}{5}$, which was improved later for $\gamma>\frac{3}{2}$ by Feireisl $[16,17]$. It should be noted that the density is allowed to vanish initially. The restriction of $\gamma$ is to show the existence of renormalized solutions introduced by DiPerna and Lions [13]. If the solution has certain symmetry, the global existence of the weak solution was obtained for any $\gamma>1$ in $[25,36]$. Hoff [19] also obtained the global existence of weak solutions in case $\gamma \geq 1$ if the initial density and velocity is a general small perturbation of a non-vacuum resting state.

Another issue concerns the existence of strong solutions to the compressible Navier-Stokes equations (1.1). When the density is positive initially, it turns out that the density will be away from vacuum at least locally in time. If, furthermore, the initial data are sufficient regular, there will exist a unique solution under various boundary conditions, see $[3,4,6,32]$ and reference therein. For instance, Solonnikov obtained in [35] a local existence of strong solutions with periodic non-vacuum data. However, for the general case allowing initial vanishing densities, it was shown recently in [3] that the Navier-Stokes equations admit a local strong solution as long as a suitable compatibility condition is satisfied initially. This is also true for the full compressible Navier-Stokes equations (cf. [5]). Moreover, a local classical solution is established by Kim [4] under various boundary conditions recently. The question is whether such solutions can be globally well defined. Although the result in [37] showed that the smooth solutions will blow up in finite time in general in the presence of vacuum, the detailed information on the formation of singularity is unknown. In this regard, Jiang and Fan [14] established a blowup criterion for such strong solutions only in terms of the density and its gradients. Furthermore, the full Navier-Stokes equations has a global strong solutions provided that the temperature and the density are well behaved.

In fact, Fan [14] proved the following blow-up criteria for the local strong solutions in the case of two dimensions. That is, if $2 \mu>\lambda$, then

$$
\lim _{T \rightarrow T^{*}}\left(\sup _{0 \leq t \leq T}\|\rho\|_{L^{\infty}},\left\|\rho^{-1}\right\|_{L^{\infty}},\|\theta\|_{L^{\infty}}\right)+\int_{0}^{T}\left(\|\rho\|_{W^{1, q_{0}}}+\|\nabla \rho\|_{L^{2}}^{4}\right) d t=\infty
$$

where $T^{*}<\infty$ is the maximal time of existence of a strong solution. $q_{0}>3$ is a constant. For the isentropic case, the result could be reduced to

$$
\lim _{T \rightarrow T^{*}}\left(\sup _{0 \leq t \leq T}\|\rho\|_{L^{\infty}}+\int_{0}^{T}\left(\|\rho\|_{W^{1, q_{0}}}+\|\nabla \rho\|_{L^{2}}^{4}\right) d t\right)=\infty
$$

provided $7 \mu>9 \lambda$.

In contrast to the result in [14], we first [22-24] established a blowup criterion, analogous to the Beal-Kato-Majda criterion for the ideal incompressible flows for the strong and classical solutions to the isentropic compressible flows:

$$
\lim _{T \rightarrow T^{*}} \int_{0}^{T}\|\nabla u\|_{L^{\infty}} d t=\infty
$$


provided

$$
7 \mu>\lambda \text {. }
$$

Very recently, under the same assumption (1.8), Fan etc [15] establish a blowup criterion similar to (1.7) for the non-isentropic flows, that is

$$
\lim _{T \rightarrow T^{*}}\left(\|\theta\|_{L^{\infty}\left(0, T ; L^{\infty}\right)}+\|\nabla u\|_{L^{1}\left(0, T ; L^{\infty}\right)}\right)=\infty .
$$

In this paper, when the initial density is away from vacuum, we remove the restriction (1.8) and establish an improved criterion for the strong solutions to the heat-conductive flows.

We first recall the existence results of strong solutions. It is essentially proved in [4] that if

$$
\begin{aligned}
& \inf _{x \in \Omega} \rho_{0}>0, \rho_{0} \in W^{1, q}(\Omega) \text { for some } q>3, \\
& u_{0} \in H_{0}^{1}(\Omega) \cap H^{2}(\Omega), \theta_{0} \in H^{2}(\Omega), \inf \theta_{0} \geq 0
\end{aligned}
$$

with the initial boundary conditions $(1.3)-(1.4)$, then there exist a $T_{*}>0$ and a unique strong solution $(\rho, \theta, u)$ on $\left[0, T_{*}\right]$ to the problem, such that for any $q_{0} \in(3, q)$,

$$
\begin{aligned}
& \rho \in C\left(\left[0, T_{*}\right], W^{1, q_{0}}\right), \quad \rho_{t} \in C\left(\left[0, T_{*}\right], L^{q_{0}}\right), \inf \rho>0 \\
& u \in C\left(\left[0, T_{*}\right], D_{0}^{1} \cap D^{2}\right) \cap L^{2}\left(0, T_{*} ; D^{2, q_{0}}\right) \\
& u_{t} \in L^{\infty}\left(0, T_{*} ; L^{2}\right) \cap L^{2}\left(0, T_{*} ; D_{0}^{1}\right) \\
& \theta \in C\left(\left[0, T_{*}\right] ; H^{2}\right) \cap L^{2}\left(0, T_{*} ; D^{2, q_{0}}\right), \theta \geq 0 \\
& \theta_{t} \in L^{\infty}\left(0, T_{*} ; L^{2}\right) \cap L^{2}\left(0, T_{*} ; D^{1}\right) .
\end{aligned}
$$

Now, we are ready to state our main theorem of this paper.

2. Main results. Basic assumptions: $\mu$ and $\lambda$ are assumed to satisfy a physical restriction

$$
\mu+\frac{3}{2} \lambda \geq 0, \mu>0
$$

Set

$$
\gamma=\frac{R}{c_{v}}+1 .
$$

For the initial boundary value problem, we have the following blowup criterion.

Theorem 2.1. Let $Q_{T}=(0, T) \times \Omega$. Assume that the initial data satisfy (1.10). Let $(\rho, u, \theta)$ be a strong solution of the system (1.1) - (1.3) satisfying the regularity (1.11). If $T^{*}<\infty$ is the maximal time of existence, then

$$
\lim _{T \rightarrow T^{*}} \int_{0}^{T}\left(\|\nabla u\|_{L^{\infty}}+\|\theta\|_{L^{\infty}}^{2}\right) d t=\infty
$$

REMARK 2.1. The blow up criterion (2.3) is analogous to the Beal-Kato-Majda criterion for the ideal flows. For the isentropic flow, it can be reduced to

$$
\lim _{T \rightarrow T^{*}}\|\nabla u\|_{L^{1}\left(0, T ; L^{\infty}\right)}=\infty
$$


which improves previous results [7, 22, 24] when the density is away from vacuum.

REMARK 2.2. In fact, the most difficult part is to estimate the convection term $F=\rho u_{t}+\rho u \cdot \nabla u$. The condition $7 \mu>\lambda$ used in [15, 22-24] to obtain an improved energy estimate, which is necessary to estimate $F$. In order to remove the restriction $7 \mu>\lambda$, we need to derive some new estimates. Our key observation is to establish a relationship between the convect term $F$ and $\rho|u|^{4}$, see Lemmas $3.2-3.4$ below. It is only here we require the density is away from vacuum initially only. Consequently, we successfully apply the Gronwall's inequality to derive a uniform bound of $\int_{\Omega}|\nabla \rho|^{2}+$ $|\nabla u|^{2}+|\nabla \theta|^{2}+\rho|u|^{4} d x$. This will be enough to obtain the higher order regularity of $\rho, u, \theta$.

REMARK 2.3. The above results also hold for $\Omega=R^{3}$ or $T^{3}$. Note that for the Cauchy problem, we need to assume $\left(\rho_{0}-\rho^{\infty}\right) \in W^{1, q}\left(R^{3}\right)$ and inf $\rho_{0}>0$ to keep the density away from vacuum, where $\rho^{\infty}>0$ is a constant. Then one can easily deduce that the density $\rho$ is always away from vacuum provided $\int_{0}^{T}\|\nabla u\|_{L^{\infty}} d t$ is bounded.

3. Proof of Theorem 2.1. Let $(\rho, u)$ be a strong solution described in Theorem 2.1. We assume that the opposite holds, i.e

$$
\lim _{T \rightarrow T^{*}} \int_{0}^{T}\left(\|\nabla u\|_{L^{\infty}}+\|\theta\|_{L^{\infty}}^{2}\right) d t=M_{0}<\infty .
$$

By assumption (3.1) and the conservation of mass, the upper and lower bounds of density follows immediately.

Lemma 3.1. Assume that

$$
\int_{0}^{T}\|\operatorname{divu}\|_{L^{\infty}} d t \leq C, \quad 0 \leq T<T^{*} .
$$

Then

$$
\left\|\rho, \rho^{-1}\right\|_{L^{\infty}\left(Q_{T}\right)} \leq C, \quad 0 \leq T<T^{*}
$$

Proof. It follows from the conservation of mass that for $\forall q>1$,

$$
\partial_{t}\left(\rho^{q}\right)+\operatorname{div}\left(\rho^{q} u\right)+(q-1) \rho^{q} \operatorname{div} u=0,
$$

which, after integration over $\Omega$, gives

$$
\partial_{t} \int_{\Omega} \rho^{q} d x \leq(q-1)\|\operatorname{div} u\|_{L^{\infty}(\Omega)} \int_{\Omega} \rho^{q} d x
$$

that is,

$$
\partial_{t}\|\rho\|_{L^{q}} \leq \frac{q-1}{q}\|\operatorname{div} u\|_{L^{\infty}(\Omega)}\|\rho\|_{L^{q}}
$$

which implies immediately

$$
\|\rho\|_{L^{q}}(t) \leq C
$$


with $C$ independent of $q$, so the lemma follows. The same holds for $\left\|\rho^{-1}\right\|_{L^{\infty}}$.

One has the following estimate.

LEMma 3.2. It holds that for $0<t \leq T<T^{*}$

$$
\partial_{t} \int_{\Omega} \rho|u|^{4} d x \leq C\left(\|\nabla u\|_{L^{\infty}}+\|\theta\|_{L^{\infty}}^{2}+1\right)\left(\int_{\Omega} \rho|u|^{4} d x+\|\nabla u\|_{L^{2}}^{2}\right)+C .
$$

Proof. Indeed, multiplying (1.2) by $4|u|^{2} u$, and integrating over $\Omega$, we obtain by using Lemma 2.1 that

$$
\begin{aligned}
& \frac{d}{d t} \int_{\Omega} \rho|u|^{4} d x+4 \int_{\Omega}|u|^{2}\left(\mu|\nabla u|^{2}+(\lambda+\mu)(\operatorname{div} u)^{2}+2 \mu|\nabla| u||^{2}\right) d x \\
& =-4(\lambda+\mu) \int_{\Omega} u \cdot \nabla|u|^{2} \operatorname{div} u d x+4 \int_{\Omega} \operatorname{div}\left(|u|^{2} u\right) P d x \\
& \leq C \int_{\Omega}|u|^{2}|\nabla u|^{2} d x+C \int_{\Omega} \rho \theta^{2}|u|^{2} d x \\
& \leq C\|\nabla u\|_{L^{\infty}}\left(\int_{\Omega} \rho|u|^{4} d x+\|\nabla u\|_{L^{2}}^{2}\right)+C\|\theta\|_{L^{\infty}}^{2}
\end{aligned}
$$

where in the last inequality we have used the following simple fact due to (3.3)

$$
\begin{aligned}
\int_{\Omega}|u|^{2}|\nabla u|^{2} d x & \leq C \int_{\Omega} \rho^{\frac{1}{2}}|u|^{2}|\nabla u|^{2} d x \\
& \leq C\left(\int_{\Omega} \rho|u|^{4} d x\right)^{\frac{1}{2}}\|\nabla u\|_{L^{4}}^{2} \\
& \leq C\left(\int_{\Omega} \rho|u|^{4} d x\right)^{\frac{1}{2}}\|\nabla u\|_{L^{2}}\|\nabla u\|_{L^{\infty}} \\
& \leq C\|\nabla u\|_{L^{\infty}}\left(\int_{\Omega} \rho|u|^{4} d x+\|\nabla u\|_{L^{2}}^{2}\right) .
\end{aligned}
$$

This completes the proof of Lemma 3.2 .

Next, we have $\theta \geq 0$ in $[0, T] \times \Omega$. The proof is standard, one can refer to [16] for more detail (see also [15]).

Now, we are ready to bound the first-order spatial derivatives of $\rho$ and $u$.

LEMMA 3.3. It holds for any $0<T<T^{*}$,

$$
\begin{aligned}
& \frac{d}{d t} \int_{\Omega}\left(\frac{C}{\kappa} \rho \theta^{2}+\mu|\nabla u|^{2}+(\lambda+\mu)(\operatorname{div} u)^{2}\right) d x+C\|\nabla \theta\|_{L^{2}}^{2}+\int_{\Omega} \rho u_{t}^{2} d x \\
& \leq C\left(\|\theta\|_{L^{\infty}}^{2}+\|\nabla u\|_{L^{\infty}}+1\right)\left(\int_{\Omega}\left(\rho|u|^{4}+\rho \theta^{2}\right) d x+\|\nabla u\|_{L^{2}}^{2}+\|\nabla \rho\|_{L^{2}}^{2}\right)+C .
\end{aligned}
$$

Proof. Multiplying the momentum equation by $u_{t}$, we get after integration by parts that

$$
\begin{aligned}
& \frac{d}{d t} \int_{\Omega}\left(\frac{\mu}{2}|\nabla u|^{2}+\frac{\lambda+\mu}{2}(\operatorname{div} u)^{2}\right) d x+\int_{\Omega} \rho u_{t}^{2} d x \\
& =-\int_{\Omega} \rho u \cdot \nabla u \cdot u_{t} d x-\int_{\Omega} u_{t} \cdot \nabla P d x .
\end{aligned}
$$


We easily derive that

$$
\begin{aligned}
& \left|\int_{\Omega} \rho u \cdot \nabla u \cdot u_{t} d x\right| \\
& \leq \frac{1}{4} \int_{\Omega} \rho u_{t}^{2} d x+\int_{\Omega} \rho|u \cdot \nabla u|^{2} d x \\
& \leq \frac{1}{4} \int_{\Omega} \rho u_{t}^{2} d x+C\|\nabla u\|_{L^{\infty}}\left(\int_{\Omega} \rho|u|^{4} d x+\|\nabla u\|_{L^{2}}^{2}\right) .
\end{aligned}
$$

For the last term of the righthand side of (3.8),

$$
\begin{aligned}
\left|\int_{\Omega} u_{t} \cdot \nabla P d x\right| & \leq C\left\|\rho^{1 / 2} u_{t}\right\|_{L^{2}}\left(\|\theta\|_{L^{\infty}}\|\nabla \rho\|_{L^{2}}+\|\nabla \theta\|_{L^{2}}\right) \\
& \leq \frac{1}{4} \int_{\Omega} \rho u_{t}^{2} d x+C\left(\|\theta\|_{L^{\infty}}^{2}\|\nabla \rho\|_{L^{2}}^{2}+\|\nabla \theta\|_{L^{2}}^{2}\right),
\end{aligned}
$$

which, together with (3.8) and (3.9), yields that

$$
\begin{aligned}
& \frac{d}{d t} \int_{\Omega} \mu|\nabla u|^{2}+(\lambda+\mu)(\operatorname{div} u)^{2} d x+\int_{\Omega} \rho u_{t}^{2} d x \\
& \leq C\left(\|\theta\|_{L^{\infty}}^{2}+\|\nabla u\|_{L^{\infty}}\right)\left(\int_{\Omega} \rho|u|^{4} d x+\|\nabla u\|_{L^{2}}^{2}+\|\nabla \rho\|_{L^{2}}^{2}\right)+C\|\nabla \theta\|_{L^{2}}^{2} .
\end{aligned}
$$

Multiplying $\theta$ on both sides of the energy equations, one has

$$
\frac{1}{2} \frac{d}{d t} \int_{\Omega} \rho \theta^{2} d x+\kappa \int_{\Omega}|\nabla \theta|^{2} d x \leq C\|\operatorname{div} u\|_{L^{\infty}} \int_{\Omega} \rho \theta^{2} d x+C\|\theta\|_{L^{\infty}}\|\nabla u\|_{L^{2}}^{2} .
$$

Adding (3.12) multiplied by $2 C / \kappa$ to (3.11) gives (3.7).

We are now ready to show the desired $L^{\infty}\left(0, T ; L^{2}(\Omega)\right)$ estimate of $\nabla \rho$ and $\nabla u$.

Lemma 3.4. Under the assumption (3.1), it holds that for $0 \leq T<T^{*}$,

$$
\begin{gathered}
\sup _{0 \leq t \leq T} \int_{\Omega} \rho \theta^{2}+|\nabla \rho|^{2}+|\nabla u|^{2}+\rho|u|^{4} d x+\int_{0}^{T} \int_{\Omega} \rho u_{t}^{2}+|\nabla \theta|^{2} d x d t \leq C, \\
\int_{0}^{T}\|\nabla u\|_{H^{1}(\Omega)}^{2} d t \leq C, \quad 0 \leq T<T^{*} .
\end{gathered}
$$

Proof. Applying $\nabla$ on both sides of the mass equation and multiplying the resulting equation by $2 \nabla \rho$, we obtain

$$
\partial_{t}\left(|\nabla \rho|^{2}\right)+\operatorname{div}\left(|\nabla \rho|^{2} u\right)+|\nabla \rho|^{2} \operatorname{div} u+2(\nabla \rho)^{t} \nabla u \nabla \rho+2 \rho \nabla \rho \cdot \nabla \operatorname{div} u=0,
$$

which, after integration over $\Omega$ by parts, gives

$$
\partial_{t}\|\nabla \rho\|_{L^{2}}^{2} \leq C(\varepsilon)\left(\|\nabla u\|_{L^{\infty}}+1\right)\|\nabla \rho\|_{L^{2}}^{2}+\varepsilon\left\|\nabla^{2} u\right\|_{L^{2}}^{2} .
$$

On the other hand, since $u$ is a solution of the elliptic system

$$
\mu \triangle u+(\lambda+\mu) \nabla \operatorname{div} u=\rho u_{t}+\rho u \cdot \nabla u+\nabla P
$$


it follows from the classical regularity theory that

$$
\begin{aligned}
\left\|\nabla^{2} u\right\|_{L^{2}} & \leq C\left\|\rho u_{t}\right\|_{L^{2}}+C\|u \cdot \nabla u\|_{L^{2}}+C\|\nabla P\|_{L^{2}} \\
& \leq C\left(\left\|\rho u_{t}\right\|_{L^{2}}+\|\nabla u\|_{L^{2}}^{3 / 2}\left\|\nabla^{2} u\right\|_{L^{2}}^{1 / 2}+\|\theta\|_{L^{\infty}}\|\nabla \rho\|_{L^{2}}+\|\nabla \theta\|_{L^{2}}\right),
\end{aligned}
$$

which implies

$$
\left\|\nabla^{2} u\right\|_{L^{2}} \leq C\left(\left\|\rho u_{t}\right\|_{L^{2}}+\|\nabla u\|_{L^{2}}^{2}+\|\theta\|_{L^{\infty}}\|\nabla \rho\|_{L^{2}}+\|\nabla \theta\|_{L^{2}}\right) .
$$

We deduce from (3.7) (3.15) and (3.16) by choosing $\varepsilon$ small enough then applying Gronwall's inequality that (3.13) holds. Estimate (3.14) is a simple consequence of (3.13) and (3.16).

Next, we will derive higher derivatives based on the previous estimates.

Lemma 3.5. Under the assumption (3.1), it holds that for $0 \leq T<T^{*}$,

$$
\begin{aligned}
& \sup _{0 \leq t \leq T}\left(\|\nabla \theta\|_{L^{2}}^{2}+\left\|u_{t}\right\|_{L^{2}}^{2}\right) \\
& \quad+\int_{0}^{T}\left(\left\|\theta_{t}\right\|_{L^{2}}^{2}+\left\|\nabla u_{t}\right\|_{L^{2}}^{2}+\left\|\nabla^{2} \theta\right\|_{L^{2}}^{2}+\left\|\nabla^{2} u\right\|_{L^{2}}^{4}\right) d t \leq C .
\end{aligned}
$$

Proof. Observing that

$$
\begin{aligned}
\left\|\nabla^{2} \theta\right\|_{L^{2}} & \leq C\left(\left\|\rho^{\frac{1}{2}} \theta_{t}\right\|_{L^{2}}+\|\rho u \cdot \nabla \theta\|_{L^{2}}+\|\nabla u\|_{L^{4}}^{2}\right) \\
& \leq C\left(\left\|\rho^{\frac{1}{2}} \theta_{t}\right\|_{L^{2}}+\|\nabla \theta\|_{L^{3}}+\|\nabla u\|_{L^{4}}^{2}\right) \\
& \leq C\left(\left\|\rho^{\frac{1}{2}} \theta_{t}\right\|_{L^{2}}+\|\nabla \theta\|_{L^{2}}^{\frac{1}{2}}\left\|\nabla^{2} \theta\right\|_{L^{2}}^{\frac{1}{2}}+\|\nabla u\|_{L^{4}}^{2}\right),
\end{aligned}
$$

one has

$$
\left\|\nabla^{2} \theta\right\|_{L^{2}} \leq C\left(\left\|\rho^{\frac{1}{2}} \theta_{t}\right\|_{L^{2}}+\|\nabla \theta\|_{L^{2}}+\|\nabla u\|_{L^{4}}^{2}\right) .
$$

It follows from (3.16) and (3.13) that

$$
\begin{aligned}
\|\nabla u\|_{L^{4}}^{2} \leq & C\left\|\nabla^{2} u\right\|_{L^{2}}^{3 / 2} \\
\leq & C\left\|\nabla^{2} u\right\|_{L^{2}}^{1 / 2}\left(\left\|\rho^{\frac{1}{2}} u_{t}\right\|_{L^{2}}+\|\theta\|_{L^{\infty}}+\|\nabla \theta\|_{L^{2}}\right) \\
\leq & C\left\|\nabla^{2} u\right\|_{L^{2}}^{1 / 2}\left(\left\|\rho^{\frac{1}{2}} u_{t}\right\|_{L^{2}}+\|\nabla \theta\|_{L^{2}}^{1 / 2}\left\|\nabla^{2} \theta\right\|_{L^{2}}^{1 / 2}+\|\nabla \theta\|_{L^{2}}\right) \\
\leq & C(\varepsilon)\left\|\nabla^{2} u\right\|_{L^{2}}\left(\left\|\rho^{\frac{1}{2}} u_{t}\right\|_{L^{2}}+\|\nabla \theta\|_{L^{2}}\right)+\varepsilon\left\|\nabla^{2} \theta\right\|_{L^{2}}+C \\
\leq & C(\varepsilon)\left\|\nabla^{2} u\right\|_{L^{2}}\left(\left\|\rho^{\frac{1}{2}} u_{t}\right\|_{L^{2}}+\|\nabla \theta\|_{L^{2}}\right)+C \varepsilon\left\|\rho^{\frac{1}{2}} \theta_{t}\right\|_{L^{2}} \\
& +C \varepsilon\|\nabla \theta\|_{L^{2}}+C \varepsilon\|\nabla u\|_{L^{4}}^{2}+C,
\end{aligned}
$$

which implies by choosing $\varepsilon$ small enough that

$$
\begin{aligned}
\|\nabla u\|_{L^{4}}^{2} \leq & C(\varepsilon)\left\|\nabla^{2} u\right\|_{L^{2}}\left(\left\|\rho^{\frac{1}{2}} u_{t}\right\|_{L^{2}}+\|\nabla \theta\|_{L^{2}}\right) \\
& +C \varepsilon\left\|\rho^{\frac{1}{2}} \theta_{t}\right\|_{L^{2}}+C\|\nabla \theta\|_{L^{2}}+C .
\end{aligned}
$$

This, together with (3.19), yields

$$
\left\|\nabla^{2} \theta\right\|_{L^{2}}^{2} \leq C\left\|\rho^{\frac{1}{2}} \theta_{t}\right\|_{L^{2}}^{2}+C\left(\left\|\nabla^{2} u\right\|_{L^{2}}^{2}+1\right)\left(\left\|\rho^{\frac{1}{2}} u_{t}\right\|_{L^{2}}^{2}+\|\nabla \theta\|_{L^{2}}^{2}\right)+C .
$$


Multiplying the energy equation by $\theta_{t}$ in $L^{2}(\Omega)$, we use (2.13) to conclude

$$
\begin{aligned}
\frac{k}{2} & \frac{d}{d t} \int_{\Omega}|\nabla \theta|^{2} d x+c_{v} \int_{\Omega} \rho \theta_{t}^{2} d x \\
= & -c_{v} \int_{\Omega} \rho(u \cdot \nabla) \theta \theta_{t} d x-\int_{\Omega} P \operatorname{div} u \theta_{t} d x+\int_{\Omega}\left(\frac{\mu}{2}\left(\nabla u+\nabla u^{t}\right)^{2}+\lambda(\operatorname{div} u)^{2}\right) \theta_{t} d x \\
\leq & C\|u\|_{L^{\infty}}\|\nabla \theta\|_{L^{2}}\left\|\rho^{\frac{1}{2}} \theta_{t}\right\|_{L^{2}}+C\|\nabla u\|_{L^{2}}\left\|\rho^{\frac{1}{2}} \theta_{t}\right\|_{L^{2}}+C\|\nabla u\|_{L^{4}}^{2}\left\|\rho^{\frac{1}{2}} \theta_{t}\right\|_{L^{2}} \\
\leq & C\|\nabla u\|_{H^{1}}\|\nabla \theta\|_{L^{2}}\left\|\rho^{\frac{1}{2}} \theta_{t}\right\|_{L^{2}}+C(\varepsilon)\left(\left\|\nabla^{2} u\right\|_{L^{2}}^{2}+1\right)\left(\left\|\rho^{\frac{1}{2}} u_{t}\right\|_{L^{2}}^{2}+\|\nabla \theta\|_{L^{2}}^{2}\right) \\
& +C \varepsilon \int_{\Omega} \rho \theta_{t}^{2} d x+C \\
\leq & C \varepsilon \int_{\Omega} \rho \theta_{t}^{2} d x+C(\varepsilon)\left(\left\|\nabla^{2} u\right\|_{L^{2}}^{2}+1\right)\left(\left\|\rho^{\frac{1}{2}} u_{t}\right\|_{L^{2}}^{2}+\|\nabla \theta\|_{L^{2}}^{2}\right)+C,
\end{aligned}
$$

which as well as (3.22) gives

$$
\begin{aligned}
& C \kappa \frac{d}{d t} \int_{\Omega}|\nabla \theta|^{2} d x+C c_{v} \int_{\Omega} \rho \theta_{t}^{2} d x+\left\|\nabla^{2} \theta\right\|_{L^{2}}^{2} \\
& \leq C\left(\left\|\nabla^{2} u\right\|_{L^{2}}^{2}+1\right)\left(\left\|\rho^{\frac{1}{2}} u_{t}\right\|_{L^{2}}^{2}+\|\nabla \theta\|_{L^{2}}^{2}\right)+C .
\end{aligned}
$$

Taking $\partial_{t}$ to the momentum equation, multiplying the resulting equation by $u_{t}$, integrating over $\Omega$, we find that

$$
\begin{aligned}
& \frac{1}{2} \frac{d}{d t} \int_{\Omega} \rho u_{t}^{2} d x+\int_{\Omega}\left(\mu\left|\nabla u_{t}\right|^{2}+(\lambda+\mu)\left(\operatorname{div} u_{t}\right)^{2}\right) d x \\
& =\int_{\Omega} P_{t} \operatorname{div} u_{t} d x-\int_{\Omega} \rho u \cdot \nabla\left[\left(u_{t}+u \cdot \nabla u\right) u_{t}\right] d x-\int_{\Omega} \rho u_{t} \cdot \nabla u \cdot u_{t} d x \\
& =I_{1}+I_{2}+I_{3} .
\end{aligned}
$$

Observing that $\left|P_{t}\right| \leq C\left|\rho_{t}\right| \theta+\left|\theta_{t}\right|$, and

$$
\begin{aligned}
& \left\|\rho_{t}\right\|_{L^{2}} \leq\left(\|u \cdot \nabla \rho\|_{L^{2}}+\|\rho \operatorname{div} u\|_{L^{2}}\right) \\
& \quad \leq C\left(1+\|u\|_{L^{\infty}}\right) \leq C\left(1+\left\|\nabla^{2} u\right\|_{L^{2}}^{\frac{1}{2}}\right),
\end{aligned}
$$

we can estimate $I_{1}$ as follows:

$$
\begin{aligned}
\left|I_{1}\right| \leq C & \left\|\nabla u_{t}\right\|_{L^{2}}\left(\|\theta\|_{L^{\infty}}+\|\nabla \theta\|_{L^{2}}^{1 / 2}\left\|\nabla^{2} \theta\right\|_{L^{2}}^{1 / 2}\left\|\nabla^{2} u\right\|_{L^{2}}^{1 / 2}+\left\|\rho^{1 / 2} \theta_{t}\right\|_{L^{2}}\right) \\
\leq \varepsilon & \left(\left\|\nabla u_{t}\right\|_{L^{2}}^{2}+\left\|\nabla^{2} \theta\right\|_{L^{2}}^{2}\right)+C(\varepsilon)\|\theta\|_{L^{\infty}}^{2}+C(\varepsilon)\left\|\nabla^{2} u\right\|_{L^{2}}^{2}\|\nabla \theta\|_{L^{2}}^{2} \\
& +C(\varepsilon)\left\|\rho^{1 / 2} \theta_{t}\right\|_{L^{2}}^{2} .
\end{aligned}
$$

For $I_{2}$, we have

$$
\begin{aligned}
\left|I_{2}\right| \leq & \int_{\Omega} \rho\left|u \| u_{t}\right|\left|\nabla u_{t}\right| d x+\int_{\Omega} \rho|u||\nabla u|^{2}\left|u_{t}\right| d x \\
& +\int_{\Omega} \rho|u|^{2}\left|\nabla^{2} u\right|\left|u_{t}\right| d x+\int_{\Omega} \rho\left|u\|\nabla u\| \nabla u_{t}\right| d x \\
\leq & C\|\nabla u\|_{L^{2}}\left\|\nabla u_{t}\right\|_{L^{2}}\left\|\rho^{1 / 2} u_{t}\right\|_{L^{2}}^{1 / 2}\left\|u_{t}\right\|_{L^{6}}^{1 / 2}+C\|u\|_{L^{6}}\|\nabla u\|_{L^{2}}\|\nabla u\|_{L^{6}}\left\|u_{t}\right\|_{L^{6}} \\
& +C\|\nabla u\|_{L^{2}}^{2}\left\|\nabla^{2} u\right\|_{L^{2}}\left\|u_{t}\right\|_{L^{6}}+C\|\nabla u\|_{L^{2}}^{2}\|\nabla u\|_{H^{1}}\left\|\nabla u_{t}\right\|_{L^{2}} \\
\leq & \varepsilon\left\|\nabla u_{t}\right\|_{L^{2}}^{2}+C(\varepsilon)\left\|\rho^{1 / 2} u_{t}\right\|_{L^{2}}^{2}+C(\varepsilon)\|\nabla u\|_{H^{1}}^{2}
\end{aligned}
$$


Finally, we bound $I_{3}$,

$$
\begin{aligned}
\left|I_{3}\right| & \leq C\|\nabla u\|_{L^{2}}\left\|\rho^{1 / 2} u_{t}\right\|_{L^{4}}^{2} \\
& \leq C\left\|\rho^{1 / 2} u_{t}\right\|_{L^{6}}^{3 / 2}\left\|\rho^{1 / 2} u_{t}\right\|_{L^{2}}^{1 / 2} \\
& \leq \varepsilon\left\|\nabla u_{t}\right\|_{L^{2}}^{2}+C \varepsilon^{-1}\left\|\rho^{1 / 2} u_{t}\right\|_{L^{2}}^{2} .
\end{aligned}
$$

Substituting (3.26)-(3.28) into (3.24), and taking $\varepsilon$ small enough, we arrive at

$$
\begin{aligned}
& \frac{d}{d t} \int_{\Omega} \rho u_{t}^{2} d x+\int_{\Omega}\left(\mu\left|\nabla u_{t}\right|^{2}+(\lambda+\mu)\left(\operatorname{div} u_{t}\right)^{2}\right) d x \\
& \leq C\left(\left\|\nabla^{2} u\right\|_{L^{2}}^{2}+1\right)\left(\left\|\rho^{1 / 2} u_{t}\right\|_{L^{2}}^{2}+\|\nabla \theta\|_{L^{2}}^{2}+1\right) \\
& \quad+C\left\|\rho^{1 / 2} \theta_{t}\right\|_{L^{2}}^{2}+C\left\|\nabla^{2} \theta\right\|_{L^{2}}^{2}+C\|\theta\|_{L^{\infty}}^{2}+C .
\end{aligned}
$$

Adding (3.29) to (3.22) multiplied by some sufficiently large $C_{0}$, then applying Gronwall's inequality, we finally obtain

$$
\sup _{0 \leq t \leq T}\left(\|\nabla \theta\|_{L^{2}}^{2}+\left\|u_{t}\right\|_{L^{2}}^{2}\right)+\int_{0}^{T}\left(\left\|\theta_{t}\right\|_{L^{2}}^{2}+\left\|\nabla u_{t}\right\|_{L^{2}}^{2}+\left\|\nabla^{2} \theta\right\|_{L^{2}}^{2}\right) d t \leq C,
$$

which as well as (3.16) yields

$$
\int_{0}^{T}\left\|\nabla^{2} u\right\|_{L^{2}}^{4} d t \leq C \int_{0}^{T}\left(1+\|\theta\|_{L^{\infty}}^{2}\right)^{2} d t \leq C \int_{0}^{T}\left(1+\left\|\nabla^{2} \theta\right\|_{L^{2}}\right)^{2} d t \leq C .
$$

Next, we will derive the desired estimates for $\theta_{t}$. In fact, we have

Lemma 3.6. For any $T<T^{*}$, it holds that

$$
\begin{gathered}
\sup _{0 \leq t \leq T} \int_{\Omega} \rho \theta_{t}^{2} d x+\int_{0}^{T}\left\|\nabla \theta_{t}\right\|_{L^{2}}^{2} d t \leq C \\
\sup _{0 \leq t \leq T}\|\nabla \theta\|_{H^{1}}^{2} \leq C .
\end{gathered}
$$

Taking $\partial_{t}$ on both sides of the energy equation, then multiplying the resulting equation by $\theta_{t}$ in $L^{2}(\Omega)$, we obtain

$$
\begin{aligned}
& \frac{1}{2} \frac{d}{d t} \int_{\Omega} \rho \theta_{t}^{2} d x+\kappa \int_{\Omega}\left|\nabla \theta_{t}\right|^{2} d x \\
& =\int_{\Omega} R \rho \theta_{t}^{2} \operatorname{div} u d x+\int_{\Omega} R \rho_{t} \theta \operatorname{div} u \theta_{t} d x+\int_{\Omega} R \rho \theta \operatorname{div} u_{t} \theta_{t} d x \\
& +\int_{\Omega}\left[\mu\left(\nabla u+\nabla u^{t}\right):\left(\nabla u_{t}+\nabla u_{t}^{t}\right)+2 \lambda \operatorname{div} u \operatorname{div} u_{t}\right] \theta_{t} d x \\
& -\int_{\Omega} \rho_{t} u \cdot \nabla \theta \theta_{t} d x-\int_{\Omega} \rho u_{t} \cdot \nabla \theta \theta_{t} d x-\int_{\Omega} \rho_{t} \theta_{t}^{2} d x=\sum_{i=1}^{7} J_{i} .
\end{aligned}
$$

It is clear that

$$
\left|J_{1}\right| \leq C\left\|\rho^{1 / 2} \theta_{t}\right\|_{L^{2}}^{2}\|\nabla u\|_{L^{\infty}} .
$$


It follows from (3.25) that

$$
\begin{gathered}
\left|J_{2}\right| \leq C\left\|\rho_{t}\right\|_{L^{2}}\|\theta\|_{L^{6}}\|\nabla u\|_{L^{6}}\left\|\theta_{t}\right\|_{L^{6}} \\
\leq C\left(1+\left\|\nabla^{2} u\right\|_{L^{2}}^{1 / 2}\right)\left\|\nabla^{2} u\right\|_{L^{2}}\left\|\nabla \theta_{t}\right\|_{L^{2}} \\
\leq \varepsilon\left\|\nabla \theta_{t}\right\|_{L^{2}}^{2}+C(\varepsilon)\left\|\nabla^{2} u\right\|_{L^{2}}^{4}+C, \\
\left|J_{5}\right| \leq\left\|\rho_{t}\right\|_{L^{2}}\left\|\nabla^{2} u\right\|_{L^{2}}^{1 / 2}\left\|\nabla \theta_{t}\right\|_{L^{2}}\left\|\nabla^{2} \theta\right\|_{L^{2}}^{1 / 2} \\
\leq C\left(1+\left\|\nabla^{2} u\right\|_{L^{2}}\right)\left\|\nabla^{2} \theta\right\|_{L^{2}}^{1 / 2}\left\|\nabla \theta_{t}\right\|_{L^{2}} \\
\leq C+C\left\|\nabla^{2} u\right\|_{L^{2}}^{4}+C(\varepsilon)\left\|\nabla^{2} \theta\right\|_{L^{2}}^{2}+\varepsilon\left\|\nabla \theta_{t}\right\|_{L^{2}}^{2},
\end{gathered}
$$

and

$$
\begin{aligned}
\left|J_{7}\right| & \leq\left\|\rho_{t}\right\|_{L^{2}}\left\|\theta_{t}\right\|_{L^{2}}^{1 / 2}\left\|\nabla \theta_{t}\right\|_{L^{2}}^{3 / 2} \\
& \leq C(\varepsilon)\left(1+\left\|\nabla^{2} u\right\|_{L^{2}}^{4}\right)\left\|\rho^{1 / 2} \theta_{t}\right\|_{L^{2}}^{2}+\varepsilon\left\|\nabla \theta_{t}\right\|_{L^{2}}^{2} .
\end{aligned}
$$

The estimate of $J_{3}$ is easy.

$$
\begin{aligned}
\left|J_{3}\right| & \leq C\left\|\rho^{1 / 2} \theta_{t}\right\|_{L^{2}}\|\theta\|_{L^{\infty}}\left\|\nabla u_{t}\right\|_{L^{2}} \\
& \leq\left\|\nabla u_{t}\right\|_{L^{2}}^{2}+C\left\|\rho^{1 / 2} \theta_{t}\right\|_{L^{2}}^{2}\|\theta\|_{L^{\infty}}^{2} .
\end{aligned}
$$

Cauchy inequality yields directly that

$$
\begin{aligned}
\left|J_{4}\right| & \leq C\|\nabla u\|_{L^{6}}\left\|\nabla u_{t}\right\|_{L^{2}}\left\|\theta_{t}\right\|_{L^{3}} \\
& \leq C\left\|\nabla^{2} u\right\|_{L^{2}}\left\|\rho^{1 / 2} \theta_{t}\right\|_{L^{2}}^{\frac{1}{2}}\left\|\nabla \theta_{t}\right\|_{L^{2}}^{\frac{1}{2}}\left\|\nabla u_{t}\right\|_{L^{2}} \\
& \leq \varepsilon\left\|\nabla \theta_{t}\right\|_{L^{2}}^{2}+\varepsilon\left\|\nabla u_{t}\right\|_{L^{2}}^{2}+C(\varepsilon)\left(\left\|\rho^{1 / 2} \theta_{t}\right\|_{L^{2}}^{2}\left\|\nabla u_{t}\right\|_{L^{2}}^{2}+\left\|\nabla^{2} u\right\|_{L^{2}}^{4}\right),
\end{aligned}
$$

and

$$
\left|J_{6}\right| \leq C\left\|\rho u_{t}\right\|_{L^{2}}\|\nabla \theta\|_{L^{3}}\left\|\nabla \theta_{t}\right\|_{L^{2}} \leq \varepsilon\left\|\nabla \theta_{t}\right\|_{L^{2}}^{2}+C \varepsilon^{-1}\left\|\nabla^{2} \theta\right\|_{L^{2}} .
$$

Substituting (3.33) - (3.39) into (3.32), we integrate to obtain for small $\varepsilon$ that

$$
\left\|\rho^{1 / 2} \theta_{t}\right\|_{L^{2}}^{2}+\left\|\nabla \theta_{t}\right\|_{L^{2}\left(0, T ; L^{2}\right)}^{2} \leq C+C \int_{0}^{t}\left(1+\|\nabla u\|_{H^{1}}^{2}\right)\left\|\rho^{1 / 2} \theta_{t}\right\|_{L^{2}}^{2} d s, \quad 0 \leq t \leq T,
$$

which, by applying Gronwall's inequality, implies (3.30). As a consequence of (3.30), we see that $\kappa \Delta \theta$ from the energy equation is bounded by a constant, which implies (3.31) immediately.

Finally, we derive the $L^{q}$ bounds of the density.

Lemma 3.7. Let $q$ be the same as in (1.10). Then

$$
\begin{gathered}
\sup _{0 \leq t \leq T}\|\nabla \rho\|_{L^{q}} \leq C \\
\int_{0}^{T}\left(\|\nabla u\|_{W^{1, q}}^{2}+\|\nabla \theta\|_{W^{1, q}}^{2}\right) d t \leq C .
\end{gathered}
$$


Proof. Differentiating (1.1) with respect to $x_{j}$ and multiplying the resulting equation by $|\nabla \rho|^{q-2} \partial_{j} \rho$, one deduces that

$$
\begin{aligned}
\frac{d}{d t} \int_{\Omega}|\nabla \rho|^{q} d x & \leq C \int_{\Omega}\left(\left|\nabla u\left\|\left.\nabla \rho\right|^{q}+\left|\rho\left\|\left.\nabla \rho\right|^{q-1}\right\| \nabla^{2} u\right|\right) d x\right.\right. \\
& \leq C\|\nabla u\|_{L^{\infty}}\|\nabla \rho\|_{L^{q}}^{q}+C\left\|\nabla^{2} u\right\|_{L^{q}}\|\nabla \rho\|_{L^{q}}^{q-1},
\end{aligned}
$$

Using the regularity of elliptic equations again, we have

$$
\begin{aligned}
\|u\|_{W^{1, q}} & \leq C\left(\left\|u_{t}\right\|_{L^{q}}+\|u \cdot \nabla u\|_{L^{q}}+\|\nabla \rho\|_{L^{q}}+\|\nabla \theta\|_{L^{q}}\right) \\
& \leq C\left(\left\|\nabla u_{t}\right\|_{L^{2}}+\|\nabla u\|_{H^{1}}^{2}+\|\nabla \rho\|_{L^{q}}+\|\nabla \theta\|_{H^{1}}\right) .
\end{aligned}
$$

Integrating the above inequality over $(0, T)$ and make use of Gronwall's inequality to show that

$$
\begin{gathered}
\sup _{0 \leq t \leq T}\|\nabla \rho\|_{L^{q}} \leq C \\
\int_{0}^{T}\left(\|\nabla u\|_{W^{1, q}}^{2}+\|\nabla \theta\|_{W^{1, q}}^{2}\right) d t \leq C .
\end{gathered}
$$

The proof is finished.

We are now ready to extend the strong solutions beyond the time $T^{*}$.

In fact, in view of Lemma $3.4-3.7$, the functions $\left.(\rho, u, \theta)\right|_{t=T^{*}}=\lim _{t \rightarrow T^{*}}(\rho, u, \theta)$ satisfy the conditions imposed on the initial data (1.10) at the time $t=T^{*}$. Therefore, we can take $\left.(\rho, u, \theta)\right|_{t=T^{*}}$ as the initial data and apply the local existence theorem [3] to extend the strong solution beyond $T^{*}$. This contradicts the assumption on $T^{*}$.

Acknowledgement. This work is supported by Zheng Ge Ru Foundation, Hong Kong RGC Earmarked Research Grants CUHK4042/08P, and a CUHK focus area grants when the authors are visiting the Institute of Mathematical Science of the Chinese University of Hong Kong.

\section{REFERENCES}

[1] J. T. BeAl, T. KATO, AND A. MAJDA, Remarks on the breakdown of smooth solutions for the 3-D Euler equations, Commun. Math. Phys., 94 (1984), pp. 61-66.

[2] J.-Y. Chemin And N. Masmoud, About lifespan of regular solutions of equations related to viscoelastic fluids SIAM J. Math. Anal., 33:1 (2001), pp. 84-112 (electronic).

[3] Y. Сhо, Н. J. Сhoе, AND H. KIm, Unique solvablity of the initial boundary value problems for compressible viscous fluid, J. Math. Pure. Appl., 83 (2004), pp. 243-275.

[4] Y. Cho AND H. KIM, On classical solutions of the compressible Navier-Stokes equations with nonnegative initial densities, Manuscript Math., 120 (2006), pp. 91-129.

[5] Y. Cho AND H. Kim, Existence results for viscous polytropic fluids with vacuum, J. Differential Equations, 228 (2006), pp. 377-411.

[6] H. J. Chot AND H. KIM, Strong solutions of the Navier-Stokes equations for isentropic compressible fluids, J. Differential Equations, 190 (2003), pp. 504-523.

[7] H. J. Choe AND J. Bum, Regularity of weak solutions of the compressible navier-stokes equations, J. Korean Math. Soc., 40:6 (2003), pp. 1031-1050.

[8] P. Constantin, C. Fefferman, E. S. Titi, and A. Zarnescu, Regularity of coupled twodimensional nonlinear Fokker-Planck and Navier-Stokes systems, Commun. Math. Phys., 270:3 (2007), pp. 789-811.

[9] P. Constantin, C. Fefferman and A. J. Majda, Geometric constraints on potentially singular solutions for the 3-D Euler equations, Comm. Partial Differential Equations, 21 (1996), pp. 559-571. 
[10] P. Constantin and C. Fefferman, Direction of vorticity and the problem of global regularity for the Navier-Stokes equations, Indiana Univ. Math. J., 42 (1993), pp. 775-789.

[11] P. Constantin, Nonlinear inviscid incompressible dynamics, Phys. D, 86 (1995), pp. 212-219.

[12] B. Desjardins, Regularity of weak solutions of the compressible isentropic Navier-Stokes equations, Comm. Partial Differential Equations, 22 (1997), pp. 977-1008.

[13] R. J. DiPerna And P.-L. Lions, Ordinary differential equations, transport theory and Sobolev spaces, Invent. Math., 98 (1989), pp. 511-547.

[14] J. FAN AND S. JiANG, Blow-Up criteria for the navier-stokes equations of compressible fluids, J. Hyper. Diff. Equa., 5:1 (2008), pp. 167-185.

[15] J. FAN, S. JIANG AND Y. OU, a blow-up criteria for compressible viscous heat-conductive flows, Ann. I. H. Poincare(2009), doi:10.1016/j.anihpc.2009.09.012.

[16] E. Feireisl, Dynamics of viscous compressible fluids, Oxford University Press, 26 (2004).

[17] E. FeIREISL, On the motion of a viscous, compressible, and heat conducting fluid, Indiana Univ. Math. J., 53 (2004), pp. 1705-1738.

[18] D. HofF, Global existence for 1D, compressible, isentropic Navier-Stokes equations with large initial data, Trans. Amer. Math. Soc., 303:1 (1987), pp. 169-181.

[19] D. HoFf, Strong convergence to global solutions for multidimensional flows of compressible, viscous fluids with polytropic equations of state and discontinuous initial data, Arch. Rational Mech. Anal., 132 (1995), pp. 1-14.

[20] D. Hoff And D. SERre, The failure of continuous dependence on initial data for the NavierStokes equations of compressible flow, SIAM J. Appl. Math., 51 (1991), pp. 887-898.

[21] D. HofF, Compressible flow in a half-space with Navier boundary conditions, J. Math. Fluid Mech., 7 (2005), pp. 315-338.

[22] X. HuAng, Some results on blowup of solutions to the compressible Navier-Stokes equations, Ph.D Thesis, The Chinese University of Hong Kong.

[23] X. Huang and Z. Xin, A Blow-up criterion for the compressible Navier-Stokes equations, arXiv:0902.2606.

[24] X. Huang And Z. Xin, A Blow-Up Criterion for Classical Solutions to the Compressible Navier-Stokes Equations, arXiv:0903.3090.

[25] S. JIANG AND P. ZHANG, On spherically symmetric solutions of the compressible isentropic Navier-Stokes equations, Comm. Math. Phys., 215 (2001), pp. 559-581.

[26] A. V. Kazhikhov and V. V. Shelukhin, Unique global solution with respect to time of initialboundary value problems for one-dimensional equations of a viscous gas, Prikl. Mat. Meh, 41 (1977), pp. 282-291.

[27] J. LeraY, Sur le mouvement d'un liquide visqueux emplissant l'espace, Acta Math, 63 (1934), pp. 193-248.

[28] P.-L. Lions, Mathematical topics in fluid mechanics, Vol. 1 The Clarendon Press Oxford University Press, 1998, 10.

[29] P.-L. Lions, Mathematical topics in fluid mechanics, Vol. 2 The Clarendon Press Oxford University Press, 1998, 10.

[30] T. LiU, Z. XIN, And T. YAng, DVacuum states for compressible flow, Discrete Contin. Dynam. Systems, 4 (1998), pp. 1-32.

[31] A. Matsumura And T. Nishida, Initial-boundary value problems for the equations of motion of compressible viscous and heat-conductive fluids, Comm. Math. Phys., 89 (1983), pp. 445464.

[32] R. Salvi and I. Straskraba, Global existence for viscous compressible fluids and their behavior as $t \rightarrow \infty$, J. Fac. Sci. Univ. Tokyo Sect. IA. Math., 40 (1993), pp. 17-51.

[33] D. Serre, Solutions faibles globales des équations de Navier-Stokes pour un fluide compressible, C. R. Acad. Sci. Paris Sér. I Math., 303 (1986), pp. 639-642.

[34] D. SERRE, Sur l'équation monodimensionnelle d'un fluide visqueux, compressible et conducteur de chaleur, C. R. Acad. Sci. Paris Sér. I Math., 303 (1986), pp. 703-706.

[35] V. A. Solonnikov, Solvability of the initial boundary value problem for the equation a viscous compressible fluid, J. Sov. Math., 14 (1980), pp. 1120-1133.

[36] W. Sun, S. Jiang, And Z. Guo, Helically symmetric solutions to the 3-D Navier-Stokes equations for compressible isentropic fluids, J. Differential Equations, 222 (2006), pp. 263296.

[37] Z. XIN, Blowup of smooth solutions to the compressible Navier-Stokes equation with compact density, Comm. Pure Appl. Math., 51 (1998), pp. 229-240. 